\title{
Design of Biofuel Supply Chains with Variable Regional Depot and Biorefinery Locations
}

\author{
Rex T. L. Ng1,2, Christos T. Maravelias*,1,2 \\ ${ }^{1}$ Department of Chemical and Biological Engineering and ${ }^{2}$ DOE Great Lakes Bioenergy Research \\ Center, University of Wisconsin-Madison, 1415 Engineering Drive, Madison, WI 53706, USA \\ *Email: christos.maravelias@wisc.edu, Tel: +1(608)-265-9026, Fax: +1(608)-262-5434
}

\begin{abstract}
We propose a multi-period mixed-integer linear programming (MILP) model for the design and operational planning of cellulosic biofuel supply chains. Specifically, the proposed MILP model accounts for biomass selection and allocation, technology selection and capacity planning at regional depots and biorefineries. Importantly, it considers the location of regional depots and biorefineries as continuous optimization decisions. We introduce reformulation methods for the calculation of the shipments and transportation distance in order to obtain a linear model. We illustrate the applicability of the proposed methods using a two mediumscale examples with realistic data.
\end{abstract}

Keywords: Cellulosic ethanol: biorefinery; mathematical programming; optimization; reformulation.

\section{Introduction}

Renewable energy can play an important role in reducing our dependence on fossil fuels, mitigating climate impacts and increasing energy security. In 2014, electricity generation from biomass accounts for $12 \%$ of all renewable energy generated in the United States [1]. In addition to heat and power generation, cellulosic biomass can be converted into biofuels (e.g., ethanol, biodiesel, "dropin" fuels, etc.) via various technologies. Based on the REmap 2030 analysis report published by the International Renewable Energy Agency [2], the total biofuel production in the United States is expected to be 39 billion gallons.

Process systems engineering (PSE) methods have been widely applied for the design and operation planning of biofuel supply chains (SCs) [3-7], as well as the design of integrated biorefineries [8-11]. Most of the proposed SC optimization model employ economic criteria and consider a single period $[12,13]$. The introduction of multi-period models allows the modeling of seasonal biomass availability and biomass deterioration [14-16]. Furthermore, environmental [17,18] and social considerations $[19,20]$ have been studied. Finally, several approaches have been proposed for the optimization of biofuel SCs under uncertainty; for example, stochastic programming [21,22], chance constraint programming [23], and robust optimization [24] methods. 
Two aspects that have received limited attention are (1) the consideration of local depots for biomass densification and/or pretreatment, and (2) the selection of the location of depots and biorefineries. Specifically, most previous approaches either neglect the installation of depots or consider depots that are co-located with harvesting sites and, furthermore, are based on the assumption that biorefineries can only be installed at predefined locations that meet certain criteria (e.g., population census, transportation network, etc.) [25,26]. However, the efficiency of the biofuel SC, in terms of both cost and $\mathrm{CO}_{2}$ emissions, can be improved by the installation of depots and the optimization of depot and biorefinery location (e.g., the optimal depot location can be between two harvesting sites) [27-29]. Recently, Ng and Maravelias proposed the first optimization model to account for depot installation and variable depot location [30]. However, the proposed mixed-integer non-linear programming (MINLP) model is computationally expensive and cannot be used to address mediumand large-scale problems.

Accordingly, the goal of this paper is to propose a computationally tractable multi-period mixedinteger linear programming (MILP) model that accounts for biomass selection and allocation, technology selection and capacity planning at depots and biorefineries, inventory and shipment planning, and, importantly, variable depot and biorefinery locations. The reduction of the computational requirements is achieved through a series of approximation and reformulations.

The rest of this article is structured as follows: In Section 2, we present background on biofuel SC, a formal problem statement, and the assumptions used for the formulation of the model. In Section 3, we present a basic optimization model, while in Section 4 we describe several model enhancements. In Section 5, we present two examples to illustrate the applicability of our methods. We close in Section 6 with concluding remarks. We use lowercase Greek letters for parameters; Latin letters for variable; lowercase Latin italic letters for subscripts; and uppercase U/L in superscript for upper/lower bounds.

\section{Background}

\subsection{Cellulosic Biofuel Supply Chain}

60 Biomass feedstocks are harvested and potentially stored at harvesting sites. Biomass can be either shipped to a biorefinery or a regional depot where it is pretreated and/or densified into a stable and dense intermediate. The primary function of the regional depot is to allow the densified biomass to be transported economically over longer distance, thus improving the overall SC economics and reducing $\mathrm{CO}_{2}$ emissions [31]. Regional depots can be categorized as standard and quality depots [32]. 
densification, biomass is converted into intermediates that meet specific biorefinery needs. Pretreatment technologies that have low operating and capital costs, simple catalyst recovery and produce an intermediate that reduces processing intensity at the biorefinery can be considered [31]; e.g., ammonia fiber explosion (AFEX) [33], dilute acid [34], alkaline peroxide [35]. Pretreated and/or densified biomass can be stored at a depot before it is sent to the biorefinery. At the biorefinery, biomass and intermediate can be converted into biofuel via biochemical [36], thermochemical

$73[37,38]$ and catalytic $[39,40]$ platforms.

\section{4}

\subsection{Problem Statement}

In this work, we consider a one-year horizon divided into time periods $t \in \mathbf{T}$, though a multi-year horizons can also be considered. We are given biomass feedstocks (e.g., corn stover and switchgrass) at the harvesting site, intermediates produced at depots (e.g., AFEX-treated pellets), intermediates at the biorefinery, and a product (ethanol). Biomass can be converted to intermediates and ethanol through different technologies, including drying and densification with or without AFEX pretreatment at the depot. The unit production cost and conversion yield of all technologies are known. The locations of harvesting sites and biomass availability at each site and time period are given, while the locations of depots and biorefineries are optimization decisions. The unit costs associated with feedstock acquisition, inventory, and transportations are known. We are also given the regions within which potential biorefineries can be installed and the upper and lower bounds on ethanol demand.

Formally, the problem we consider is stated in terms of the following sets, subsets and parameters:

a) Compounds $i \in \mathbf{I}$ with unit price $\lambda_{i}$, unit inventory costs $\iota_{i}$ and fixed/variable transportation unit $\operatorname{cost} \kappa_{i}^{\mathrm{F}} / \kappa_{i}^{\mathrm{V}}$.

i. Biomass feedstocks $\mathbf{I}^{\mathrm{F}} \subset \mathbf{I}$ at harvesting sites.

ii. Intermediates $\mathbf{I}^{\mathrm{ID}} \subset \mathbf{I}$ produced at depots.

iii. Intermediates $\mathbf{I}^{\mathrm{IB}} \subset \mathbf{I}$ produced at biorefineries.

iv. Products $\mathbf{I}^{\mathrm{P}} \subset \mathbf{I}$ with minimum $\beta_{i, t}^{\mathrm{L}}$ and maximum $\beta_{i, t}^{\mathrm{U}}$ product demand.

v. By-products $\mathbf{I}^{\mathrm{B}} \subset \mathbf{I}$.

b) Harvesting sites $j \in \mathbf{J}$ with $x$-Cartesian coordinate $x_{j}, y$-Cartesian coordinate $y_{j}$, and biomass availability $\alpha_{i, j, t}$.

c) Potential depots $k \in \mathbf{K}$ with variable locations.

d) Potential biorefineries $l \in \mathbf{L}$ with variable locations within certain region.

e) Technologies $m \in \mathbf{M}$ with unit production cost $\mu_{m}$ and conversion coefficient $\eta_{i, i^{\prime}, m}$.

i. $\quad$ Pretreatment/densification technologies at depots $\mathbf{M}^{\mathrm{PD}} \subset \mathbf{M}$.

ii. Pretreatment technologies at biorefineries $\mathbf{M}^{\mathrm{PB}} \subset \mathbf{M}$. 
iii. Conversion technologies $\mathbf{M}^{\mathrm{CB}} \subset \mathbf{M}$.

102 Our goal is to determine the optimal number, capacity, and location of depots and biorefineries, as 103 well as the production, inventory, and shipment profiles of all SC nodes such that the total annual 104 cost is minimized.

\section{$105 \quad 2.3$ Assumptions}

106 It is assumed that all biomass at the harvesting site will be shipped to the depots/biorefineries if the 107 harvesting site is selected in the SC optimization model. This assumption can be easily relaxed by 108 dividing a site into multiple sub-sites and thereby allowing the selection of a fraction of the availability 109 of the site. For example, a county (a unit for which biomass availability data are typically available) 110 can be divided into rectangular or square cells (sub-sites).

111 Furthermore, we assume that the biomass from a harvesting site is sent to only one downstream 112 node, either a depot or a biorefinery; and, similarly, the intermediates from a depot are shipped to a 113 single biorefinery. While better solutions can be obtained, in theory, by shipping to multiple nodes 114 and/or continuously changing the destination of shipments coming from the same SC node, it is 115 difficult to implement such "dynamic" operation in practice [30]. Thus, the second assumption is 116 realistic.

117 It is further assumed that no biomass feedstock is stored at depots and biorefineries, and no 118 intermediates are stored at biorefineries. Rather, biomass is stored at harvesting sites only and 119 intermediates at depots only. This assumption has minimal effect because inventory unit costs and 120 material deterioration coefficients are practically independent of location, which means that the 121 solutions we obtain equivalent and representative of a family of solutions. Furthermore, in general, 122 material storage at the origin is preferred because material losses occur before transportation and 123 thus result in lower transportation cost.

\section{$124 \quad 3$ Basic Model}

125 In this section, we present a basic model for the design of biofuel SC with regional depots. We 126 introduce the following binary variables:

$127-W_{j} / W_{k} / W_{l}=1$ if harvesting site $j / \operatorname{depot} k /$ biorefinery $l$ is selected.

$128-U_{k, m} / U_{l, m}=1$ if technology $m$ at depot $k$ /biorefinery $l$ is selected.

$129 \bullet Z_{j, k} / Z_{j, l} / Z_{k, l}=1$ if transportation along arc $j \rightarrow k / j \rightarrow l / k \rightarrow l$ is selected.

130 and the following nonnegative continuous variables:

131 - $S_{i, j, t} / S_{i, k, t} / S_{i, l, t}$ : inventory level of compound $i$ at SC node $j / k / l$ at the end of period $t$.

- $\quad F_{i, j, k, t} / F_{i, j, l, t} / F_{i, k, l, t}$ : amount of compound $i$ shipped along arc $j \rightarrow k / j \rightarrow l / k \rightarrow l$ during period $t$. 
- $\quad G_{i, k, m, t}^{\mathrm{F}-\mathrm{PD}}$ : consumption of compound $i \in \mathbf{I}^{\mathrm{F}}$ by technology $m \in \mathbf{M}^{\mathrm{PD}}$ at depot $k$ during period $t$.

- $\quad P_{i, k, m, t}^{\mathrm{ID}-\mathrm{PD}}$ : production of compound $i \in \mathrm{I}^{\mathrm{ID}}$ by technology $m \in \mathbf{M}^{\mathrm{PD}}$ at depot $k$ during period $t$.

- $G_{i, l, m, t}^{\mathrm{F}-\mathrm{PB}}$ : consumption of compound $i \in \mathbf{I}^{\mathrm{F}}$ by technology $m \in \mathbf{M}^{\mathrm{PB}}$ at biorefinery $l$ during period $t$.

- $G_{i, l, m, t}^{\mathrm{IB}-\mathrm{CB}}$ : consumption of $i \in \mathrm{I}^{\mathrm{IB}}$ by technology $m \in \mathbf{M}^{\mathrm{CB}}$ at biorefinery $l$ during period $t$.

- $G_{i, l, m, t}^{\mathrm{ID}-\mathrm{PB}} / G_{i, l, m, t}^{\mathrm{ID}-\mathrm{CB}}$ : consumption of $i \in \mathrm{I}^{\mathrm{ID}}$ by technology $m \in \mathbf{M}^{\mathrm{PB}} / \mathbf{M}^{\mathrm{CB}}$ at biorefinery $l$ during period $t$.

- $\quad P_{i, l, m, t}^{\mathrm{IB}-\mathrm{PB}}$ : production of $i \in \mathrm{I}^{\mathrm{IB}}$ by technology $m \in \mathbf{M}^{\mathrm{PB}}$ at biorefinery $l$ during period $t$.

- $\quad P_{i, l, m, t}^{\mathrm{IPB}-\mathrm{CB}}$ : production of $i \in \mathbf{I}^{\mathrm{P}} / \mathbf{I}^{\mathrm{B}}$ by technology $m \in \mathbf{M}^{\mathrm{CB}}$ at biorefinery $l$ during period $t$.

- $\quad L_{i, l, t}$ : total sales of $i \in \mathbf{I}^{\mathrm{P}} \cup \mathbf{I}^{\mathrm{B}}$ from biorefinery $l$ during period $t$.

- $Q_{k, m} / Q_{l, m}$ : capacity of technology $m$ at depot $k /$ biorefinery $l$.

- $x_{k}^{*} / x_{l}^{*}: x$-Cartesian coordinate of depot $k /$ biorefinery $l$.

- $y_{k}^{*} / y_{l}^{*}: y$-Cartesian coordinate of depot $k /$ biorefinery $l$.

- $\quad D_{j, k} / D_{j, l} / D_{k, l}$ : length (distance) along arc $j \rightarrow k / j \rightarrow l / k \rightarrow l$.

- $\bar{D}_{j, k} / \bar{D}_{j, l} / \bar{D}_{k, l}$ : disaggregated real variables for $D_{j, k} / D_{j, l} / D_{k, l}$.

- $\breve{D}_{j, k} / \breve{D}_{j, l} / \breve{D}_{k, l}$ : disaggregated dummy variables for $D_{j, k} / D_{j, l} / D_{k, l}$.

- $\quad \bar{D}_{k, l-j} / \breve{D}_{k, l-j}$ : disaggregated real/dummy variables for $\bar{D}_{k, l}$ with respect to harvesting site $j$.

- $\quad \bar{D}_{k, l-j, m}$ : disaggregated real variable for $\bar{D}_{k, l}$ with respect to harvesting site $j$ and technology $m$.

The constraints of the model are presented in the next subsections.

\subsection{Material Balance}

The material balance at a harvesting site, depot, and biorefinery is illustrated in Figure 1.

\subsubsection{Harvesting sites}

The biomass inventory at the harvesting site at the end of time period, $S_{i, j, t}$ is equal to the inventory at the end of the previous period after considering biomass deterioration, plus the availability of biomass, minus the shipments to other SC nodes (depots or biorefineries):

$S_{i, j, t}=S_{i, j, t-1}\left(1-\gamma_{i, t}\right)+\alpha_{i, j, t} W_{j}-\sum_{k} F_{i, j, k, t}-\sum_{l} F_{i, j, l, t}, \quad i \in \mathbf{I}^{\mathrm{F}}, j, t$

where $\gamma_{i, t}$ is material loss factor of compound $i$ during time period $t$. If $W_{j}=1$, harvesting site $j$ is selected to ship compound $i$ to depots or biorefineries (when $W_{j}=0$, biomass in harvesting site $j$ may be used for other purposes, e.g., animal feed).

\subsubsection{Depots}

The inventory of biomass at the depot at the end of time period, $S_{i, k, t}$ is given by:

$S_{i, k, t}=S_{i, k, t-1}\left(1-\gamma_{i, t}\right)+\sum_{j} F_{i, j, k, t}-\sum_{m \in \mathbf{M}^{\mathrm{PD}}} G_{i, k, m, t}^{\mathrm{F}-\mathrm{PD}}, \quad i \in \mathbf{I}^{\mathrm{F}}, k, t$

Since, there is no biomass inventory at the depot (see assumptions in Section 2.3), $S_{i, k, t}=S_{i, k, t-1}=$ 0 , and equation 2 can be simplified as follows: 
166

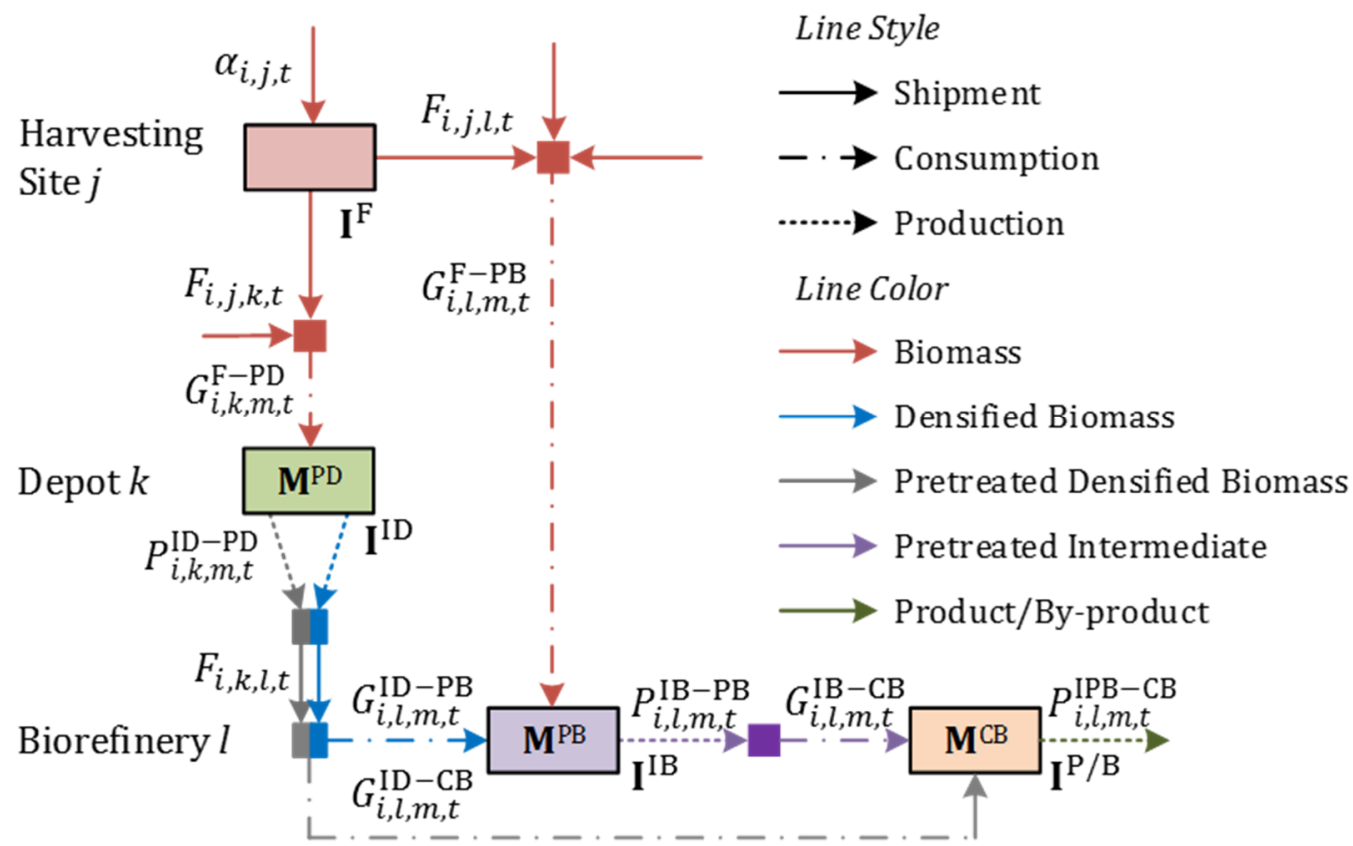

Figure 1: Material balance.

$\sum_{j} F_{i, j, k, t}=\sum_{m \in \mathbf{M}^{\mathrm{PD}}} G_{i, k, m, t}^{\mathrm{F}-\mathrm{PD}}, \quad i \in \mathbf{I}^{\mathrm{F}}, k, t$

The production $P_{i^{\prime}, k, m, t}^{\mathrm{ID}-\mathrm{PD}}$ of intermediates $i^{\prime} \in \mathbf{I}^{\mathrm{ID}}$ is determined by:

$P_{i^{\prime}, k, m, t}^{\mathrm{ID}-\mathrm{PD}}=\sum_{i \in \mathrm{I}^{\mathrm{F}}} \eta_{i, i^{\prime}, m} G_{i, k, m, t}^{\mathrm{F}-\mathrm{PD}}, \quad i^{\prime} \in \mathrm{I}^{\mathrm{ID}}, k, m \in \mathbf{M}^{\mathrm{PD}}, t$

The inventory balance of intermediates at the depot is given as:

$S_{i, k, t}=S_{i, k, t-1}\left(1-\gamma_{i, t}\right)+\sum_{m \in \mathbf{M}^{\mathrm{PD}}} P_{i, k, m, t}^{\mathrm{ID}-\mathrm{PD}}-\sum_{l} F_{i, k, l, t}, \quad i \in \mathrm{I}^{\mathrm{ID}}, k, t$

\subsubsection{Biorefineries}

Similarly, there is no biomass/intermediate inventory at the biorefinery (see assumptions in Section 2.3), which means that the mass balance of biomass and intermediate can be written as follows (see Figure 1):

$\sum_{j} F_{i, j, l, t}=\sum_{m \in \mathbf{M}^{\mathrm{PB}}} G_{i, l, m, t}^{\mathrm{F}-\mathrm{PB}}, \quad i \in \mathbf{I}^{\mathrm{F}}, l, t$

$\sum_{k} F_{i, k, l, t}=\sum_{m \in \mathbf{M}^{\mathrm{PB}}} G_{i, l, m, t}^{\mathrm{ID}-\mathrm{PB}}+\sum_{m \in \mathbf{M}^{\mathrm{CB}}} G_{i, l, m, t}^{\mathrm{ID}-\mathrm{CB}}, \quad i \in \mathbf{I}^{\mathrm{ID}}, l, t$

where biomass, $G_{i, l, m, t}^{\mathrm{F}-\mathrm{PB}}$ is sent for pretreatment; and intermediates can be split into consumption level for pretreatment technologies, $G_{i, l, m, t}^{\mathrm{ID}-\mathrm{PB}}$, and conversion technologies, $G_{i, l, m, t}^{\mathrm{ID}-\mathrm{CB}}$, respectively. For example, densified biomass from the standard $\operatorname{depot}\left(G_{i, l, m, t}^{\mathrm{ID}-\mathrm{PB}}\right)$ need to be pretreated prior to ethanol. Pretreated densified biomass produced at the quality $\operatorname{depot}\left(G_{i, l, m, t}^{\mathrm{ID}-\mathrm{CB}}\right)$ on the other hand can be sent for ethanol production directly.

For pretreatment technology $m \in \mathbf{M}^{\mathrm{PB}}$ (purple box in Figure 1), the production $P_{i^{\prime}, l, m, t}^{\mathrm{IB}-\mathrm{PB}}$ of intermediates $i^{\prime} \in \mathrm{I}^{\mathrm{IB}}$ is given by: 
$P_{i^{\prime}, l, m, t}^{\mathrm{IB}-\mathrm{PB}}=\sum_{i \in \mathbf{I}^{\mathrm{F}}} \eta_{i, i^{\prime}, m} G_{i, l, m, t}^{\mathrm{F}-\mathrm{PB}}+\sum_{i \in \mathbf{I}^{\mathrm{ID}}} \eta_{i, i^{\prime}, m} G_{i, l, m, t}^{\mathrm{ID}-\mathrm{PB}}, \quad i^{\prime} \in \mathbf{I}^{\mathrm{IB}}, l, m \in \mathbf{M}^{\mathrm{PB}}, t$

187 The consumption $G_{i, l, m, t}^{\mathrm{IB}-\mathrm{CB}}$ of intermediates $i \in \mathrm{I}^{\mathrm{IB}}$ by technologies $m \in \mathbf{M}^{\mathrm{CB}}$ is given by:

$188 \sum_{m \in \mathbf{M}^{\mathrm{CB}}} G_{i, l, m, t}^{\mathrm{IB}-\mathrm{CB}}=\sum_{m \in \mathbf{M}^{\mathrm{PB}}} P_{i, l, m, t}^{\mathrm{IB}-\mathrm{PB}}, \quad i \in \mathbf{I}^{\mathrm{IB}}, l, t$

189 For conversion technologies $m \in \mathbf{M}^{\mathrm{CB}}$ (orange box in Figure 1), all intermediates $i \in \mathbf{I}^{\mathrm{ID}} \cup \mathbf{I}^{\mathrm{IB}}$ are 190 converted into products or by-products $i^{\prime} \in \mathbf{I}^{\mathrm{P}} \cup \mathbf{I}^{\mathrm{B}}$; their production level, $P_{i^{\prime}, l, m, t}^{\mathrm{IPB}-\mathrm{CB}}$, is calculated as 191 follows:

$P_{i^{\prime}, l, m, t}^{\mathrm{IPB}-\mathrm{CB}}=\sum_{i \in \mathbf{I}^{\mathrm{ID}}} \eta_{i, i^{\prime}, m} G_{i, l, m, t}^{\mathrm{ID}-\mathrm{CB}}+\sum_{i \in \mathbf{I}^{\mathrm{IB}}} \eta_{i, i^{\prime}, m} G_{i, l, m, t}^{\mathrm{IB}-\mathrm{CB}}, \quad i^{\prime} \in \mathbf{I}^{\mathrm{P}} \cup \mathbf{I}^{\mathrm{B}}, l, m \in \mathbf{M}^{\mathrm{CB}}, t$

193 The mass balance of both products and by-products is given by:

$194 S_{i, l, t}=S_{i, l, t-1}+\sum_{m \in \mathbf{M}^{\mathrm{CB}}} P_{i, l, m, t}^{\mathrm{IPB}-\mathrm{CB}}-L_{i, l, t}, \quad i \in \mathbf{I}^{\mathrm{P}} \cup \mathbf{I}^{\mathrm{B}}, l, t$

195 The total sales, $L_{i, l, t}$, are bounded within lower and upper target levels:

$196 \beta_{i, t}^{\mathrm{L}} \leq \sum_{l} L_{i, l, t} \leq \beta_{i, t}^{\mathrm{U}}, \quad i \in \mathbf{I}^{\mathrm{P}}, t$

\section{$197 \quad 3.2$ Capacity Constraints}

198 The following constraints enforce the installation of exactly one pretreatment technology and one 199 conversion technology at the installed depots and biorefineries, respectively:

$200 \sum_{m \in \mathbf{M}^{\mathrm{PD}}} U_{k, m}=W_{k}, \quad k$

$201 \sum_{m \in \mathbf{M}^{\mathrm{CB}}} U_{l, m}=W_{l}, \quad l$

202 where $W_{k} / W_{l}$ are binary variables for the installation of depot $k /$ biorefinery $l$, and $U_{k, m} / U_{l, m}$ indicate

203 the selection of technology $m$ at $k / l$. In addition, pretreatment technology may not be necessary at 204 the biorefinery if all biomass is sent to quality depot.

$205 \sum_{m \in \mathbf{M}^{\mathrm{PB}}} U_{l, m} \leq W_{l}, \quad l$

206 The operating capacity of technology $m$ at depot $k, Q_{k, m}$ and biorefinery $l, Q_{l, m}$ are constrained as 207 follows:

$208 \epsilon_{m}^{\mathrm{L}} U_{k, m} \leq Q_{k, m} \leq \epsilon_{m}^{\mathrm{U}} U_{k, m}, \quad k, m \in \mathbf{M}^{\mathrm{PD}}$

$209 \epsilon_{m}^{\mathrm{L}} U_{l, m} \leq Q_{l, m} \leq \epsilon_{m}^{\mathrm{U}} U_{l, m}, \quad l, m \in \mathbf{M}^{\mathrm{PB}} \cup \mathbf{M}^{\mathrm{CB}}$

210 where $\epsilon_{m}^{\mathrm{L}} / \epsilon_{m}^{\mathrm{U}}$ are lower/upper bounds on capacity.

211 Consumption levels are constrained by the installed capacities, $Q_{k, m}$ and $Q_{l, m}$ as follows:

$212 \sum_{i \in \mathbf{I}^{\mathrm{F}}} G_{i, k, m, t}^{\mathrm{F}-\mathrm{PD}} \leq Q_{k, m}, \quad k, m \in \mathbf{M}^{\mathrm{PD}}, t$

$213 \sum_{i \in \mathrm{I}^{\mathrm{ID}}} G_{i, l, m, t}^{\mathrm{ID}-\mathrm{CB}}+\sum_{i \in \mathrm{I}^{\mathrm{IB}}} G_{i, l, m, t}^{\mathrm{IB}-\mathrm{CB}} \leq Q_{l, m}, \quad l, m \in \mathbf{M}^{\mathrm{CB}}, t$

$214 \quad \sum_{i \in \mathrm{I}^{\mathrm{ID}}} G_{i, l, m, t}^{\mathrm{ID}-\mathrm{PB}}+\sum_{i \in \mathrm{I}^{\mathrm{F}}} G_{i, l, m, t}^{\mathrm{F}-\mathrm{PB}} \leq Q_{l, m}, \quad l, m \in \mathbf{M}^{\mathrm{PB}}, t$ 


\subsection{Connectivity Constraints}

216 It is assumed that each harvesting site sends biomass feedstock to only one downstream node and 217 each depot sends intermediates to only one biorefinery:

$218 \quad \sum_{k} Z_{j, k}+\sum_{l} Z_{j, l}=W_{j}, \quad j$

$219 \quad \sum_{l} Z_{k, l}=W_{k}, \quad k$

220 where $Z_{j, k} / Z_{j, l} / Z_{k, l}$ are arc selection binary variables. If an arc is selected $(Z=1)$, then the 221 corresponding flows can be nonzero:

$222 \sum_{i} F_{i, j, k, t} \leq \chi_{j, k}^{\mathrm{U}} Z_{j, k}, \quad j, k, t$

$\sum_{i} F_{i, j, l, t} \leq \chi_{j, l}^{\mathrm{U}} Z_{j, l}, \quad j, l, t$

$224 \sum_{i} F_{i, k, l, t} \leq \chi_{k, l}^{\mathrm{U}} Z_{k, l}, \quad k, l, t$

225 where $\chi_{j, k}^{\mathrm{U}} / \chi_{j, l}^{\mathrm{U}} / \chi_{k, l}^{\mathrm{U}}$ are upper bounds on total shipments and can be calculated from biomass 226 availability data.

\subsection{Cyclic Inventory}

The concept of "cyclic" inventory balance is adopted to ensure the operations can be carried out in the next year and to avoid the underestimation of inventory cost. The inventory level at the beginning $(t=0)$ and end $(t=|\mathbf{T}|)$ of the horizon should be the same: $S_{i, j, t=0}=S_{i, j, t=|\mathbf{T}|}, S_{i, k, t=0}=$ $S_{i, k, t=|\mathbf{T}|}$ and $S_{i, l, t=0}=S_{i, l, t=|\mathbf{T}|}$.

\section{$232 \quad 3.5$ Harvesting Site, Depot and Biorefinery Locations}

233 Based on the Rhumb Line concept [41], the latitude and longitude of harvesting sites can be converted 234 to the $x$ - and $y$-Cartesian coordinates by projecting an oblate spheroid into a flat image. It is assumed 235 that the coordinates for harvesting sites $\left(x_{j}, y_{j}\right)$ are constants, whereas the coordinates for all depots $236\left(x_{k}^{*}, y_{k}^{*}\right)$ and biorefineries $\left(x_{l}^{*}, y_{l}^{*}\right)$ are variables. The coordinates of the depot are constrained as 237 follows:

$238 \quad x_{k}^{*} \leq x^{\mathrm{U}} W_{k}, \quad k$

$239 y_{k}^{*} \leq y^{\mathrm{U}} W_{k}, \quad k$

240 where $x^{\mathrm{U}} / y^{\mathrm{U}}$ are upper bounds of the studied region. When the depot is not selected, $x_{k}^{*}$ and $x_{k}^{*}$ will 241 return to the value of 0.

242 The location of a biorefinery at certain city/town/village can be predetermined based on population, 243 the available transportation network, and other factors. Most approaches in the literature, assume 244 that potential biorefinery locations are predefined at, for example, the centroid of the selected region 245 (gold star in Figure 2). However, a biorefinery can be installed at various points within the selected 246 region. For example, as Figure 2 shows, it may be beneficial to be installed near the border of the 
region. If the coordinates of the vertices of the region (squares in figure 2) are available, then the coordinates of the biorefinery are subject to the following convex hull constraints:

$250 y_{l}^{*}=\sum_{o} \rho_{l, o} y_{l, o}+\epsilon\left(1-W_{l}\right), \quad l$

$251 \quad \sum_{o} \rho_{l, o}=W_{l}, l$

252 where $\rho_{l, o} \in[0,1] ; x_{l, o} / y_{l, o}$ are the coordinates of the vertices (black box). Parameter $\epsilon$ can be assumed as the midpoint of the potential biorefinery region. When the potential biorefinery is not selected, $x_{l}^{*}$ and $x_{l}^{*}$ will return to the value of $\epsilon$. If the constraints defining the regions, rather than the coordinates of the vertices are available, the Quickhull Algorithm [42] can be used to calculate the coordinates of the vertices. Nonconvex regions can also be represented but at a higher computational cost.

$272 \Delta x_{j, k} \geq x_{j}-x_{k}^{*}, j, k$

$273 \Delta x_{j, k} \geq-\left(x_{j}-x_{k}^{*}\right), j, k$

\subsection{Transportation Distance}

$D_{j, k}=\Delta x_{j, k}+\Delta y_{j, k}, \quad j, k$ minimize distance):

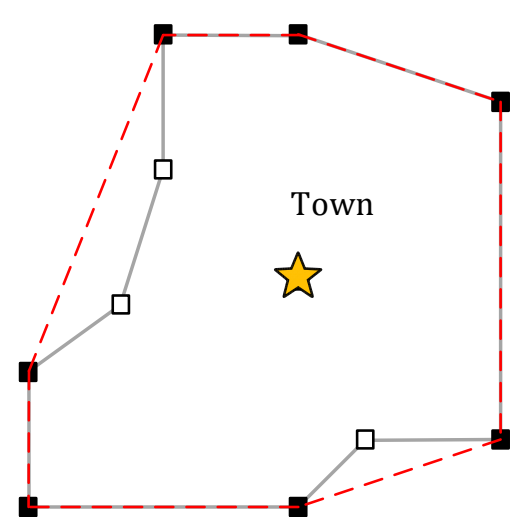

Figure 2: Area of potential biorefinery. Orange star is the coordinate of potential biorefinery assumed in previous works. Square boxes are the coordinates of the vertices defining the convex hull of the selected region (red dashed line). White boxes are the discarded interior points.

The distance between two locations can be estimated based on flow path distance, great-circle distance, Euclidean distance, rectilinear distance, etc. The rectilinear distance is a good estimation of the transportation distance in the United States as the US interstate highway and the state roadway systems are often developed based on approximate rectilinear patterns. The rectilinear distance between two locations, $D_{j, k}$ is modeled by:

where the absolute values of horizontal distance, $\Delta x_{j, k}$, and vertical distance, $\Delta y_{j, k}$ can be obtained by the following inequalities (two of which will become binding since the optimization tries to 
$275 \Delta y_{j, k} \geq-\left(y_{j}-y_{k}^{*}\right), \quad j, k$

276 Similar equations (Equations $31-35$ ) can be applied to determine $D_{j, l}$ and $D_{k, l}$. Note that positive 277 values of $D_{j, k}, D_{j, l}$, and $D_{k, l}$ are calculated even if the depot or the biorefinery are not selected.

\section{$278 \quad 3.7$ Total Annual Cost}

279 The total annual cost, TAC is calculated by:

$280 T A C=\sum_{t}\left(C_{t}^{\mathrm{FEED}}+C_{t}^{\mathrm{PROD}}+C_{t}^{\mathrm{INV}}+C_{t}^{\mathrm{TRANS}}\right)+v C^{\mathrm{CAPEX}}$

281 where $C_{t}^{\mathrm{FEED}} / C_{t}^{\mathrm{PROD}} / C_{t}^{\mathrm{INV}} / C_{t}^{\mathrm{TRANS}}$ are feedstock/production/inventory/transportation costs 282 during period $t$; $C^{\text {CAPEX }}$ is the total capital cost for depots and biorefinery installation; and $v$ is a 283 capital recovery factor.

284 The detailed formulations of the cost components are given as follows:

$285 C_{t}^{\mathrm{FEED}}=\sum_{i, j} \lambda_{i} \alpha_{i, j, t} W_{j}, \quad t$

$C_{t}^{\mathrm{PROD}}=\sum_{i, k, m} \mu_{m} G_{i, k, m, t}^{\mathrm{F}-\mathrm{PD}}+\sum_{i, l, m} \mu_{m}\left(G_{i, l, m, t}^{\mathrm{F}-\mathrm{PB}}+G_{i, l, m, t}^{\mathrm{ID}-\mathrm{PB}}+G_{i, l, m, t}^{\mathrm{ID}-\mathrm{CB}}+G_{i, l, m, t}^{\mathrm{IB}-\mathrm{CB}}\right), \quad t$

$C_{t}^{\mathrm{INV}}=\sum_{i} \iota_{i}\left(\sum_{j} S_{i, j, t}+\sum_{k} S_{i, k, t}+\sum_{l} S_{i, l, t}\right), \quad t$

$C_{t}^{\mathrm{TRANS}}=\sum_{i, j, k}\left[\left(\kappa_{i}^{\mathrm{F}}+\kappa_{i}^{\mathrm{V}} D_{j, k}\right) F_{i, j, k, t}\right]+\sum_{i, j, l}\left[\left(\kappa_{i}^{\mathrm{F}}+\kappa_{i}^{\mathrm{V}} D_{j, l}\right) F_{i, j, l, t}\right]+\sum_{i, k, l}\left[\left(\kappa_{i}^{\mathrm{F}}+\right.\right.$

$\left.\left.289 \kappa_{i}^{\mathrm{V}} D_{k, l}\right) F_{i, k, l, t}\right], t$

$C^{\mathrm{CAPEX}}=\sum_{k, m} C_{k, m}^{\mathrm{CAPEX}}+\sum_{l, m} C_{l, m}^{\mathrm{CAPEX}}$

291 where $C_{k, m}^{\mathrm{CAPEX}} / C_{l, m}^{\mathrm{CAPEX}}$ is capital cost of technology $m$ at depot $k$ /biorefinery $l$. Note that the capital 292 cost of each technology follows a nonlinear relationship to account for the economies of scale. A 293 piecewise linearization is applied to obtain these capital costs (see Supplementary Material).

\section{Model Reformulation}

\section{$295 \quad 4.1$ Approximation of Feedstock Shipment}

296 The basic formulation (Equations 1 - 35) and cost equations (Equations $36-39$, 41) in the proposed models are linear, except the bilinearities $\left(D_{j, k} F_{i, j, k, t}, D_{j, l} F_{i, j, l, t}\right.$, and $\left.D_{k, l} F_{i, k, l, t}\right)$ in the calculation of transportation cost (Equation 40). In this work, we have to consider the optimal depot and biorefinery locations which means that the depot and biorefinery locations, and therefore the distances, are variables. Nonetheless, based on the assumptions discussed in Section 2.3, we can develop an approximation and reformulation that will allow us to replace variables $F$ with constants. Specifically, since all biomass of type $i \in \mathrm{I}^{\mathrm{F}}$ from selected harvesting site $j$ is eventually shipped to a depot or biorefinery, we can replace the sum $\sum_{k, t} F_{i, j, k, t}+\sum_{l, t} F_{i, j, l, t}$ with $\sum_{t} \alpha_{i, j, t}$. The timing of the shipment can be ignored since all biomass will always be shipped. Thus, at the harvesting site, $\alpha \times D$ 
can be used to replace the terms of $F \times D$. A small error though is introduced because the actual shipments will be slightly lower than $\sum_{t} \alpha_{i, j, t}$ due to biomass deterioration during storage. An approximation of this error can be calculated prior to optimization based on some extreme inventory profiles. Specifically, for arc $j \rightarrow k / j \rightarrow l, F_{i, j, k, t}$ and $F_{i, j, l, t}$ can be replaced by the availability $\alpha_{i, j, t}$ of biomass feedstock $i \in \mathbf{I}^{\mathrm{F}}$ multiplied by correction factor $\delta_{i}$ (see Figure 3), which represents the ratio of output (biomass shipment to the depot/biorefinery) over input (total availability of biomass).

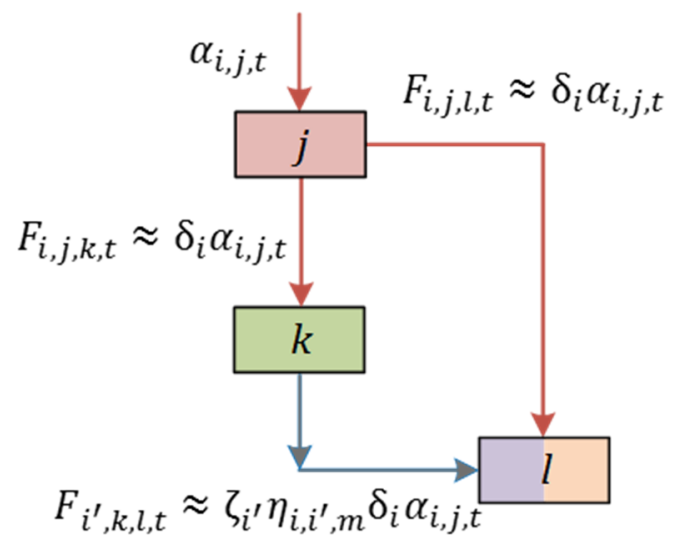

312 Figure 3: Approximation of amount of feedstock shipment

(a) Shipment at $t=1 \& 2$

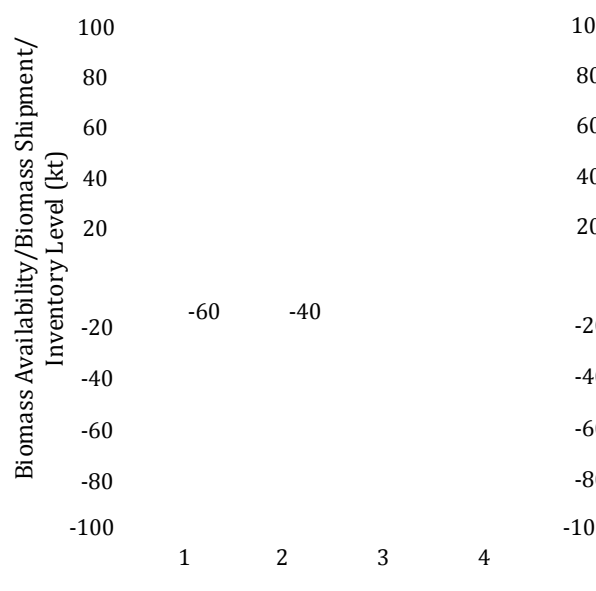

(b) Shipment at $t=4$

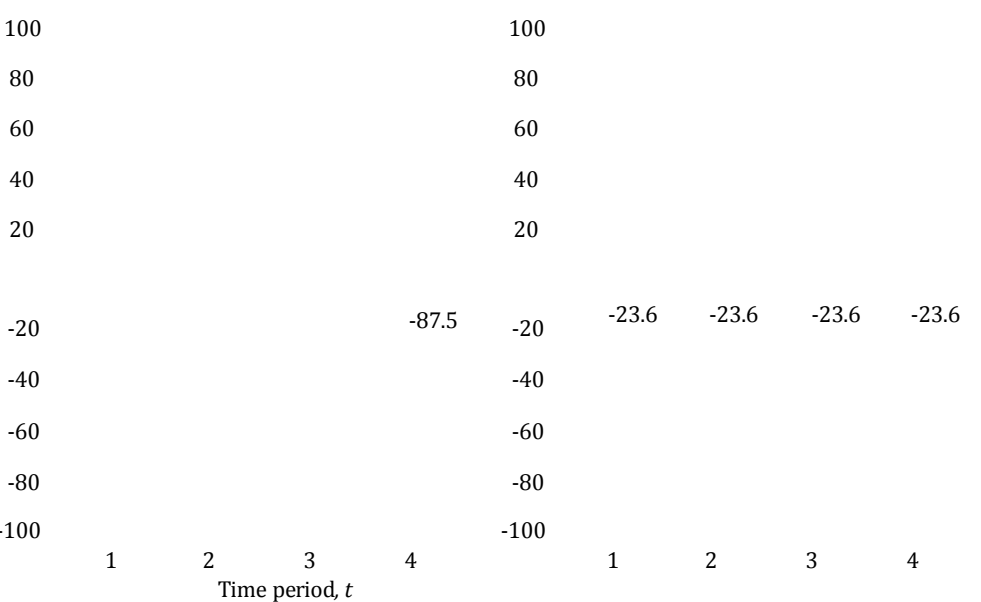

Figure 4: Harvested biomass and shipment. Positive bar indicates biomass availability; negative bar indicates biomass shipment. Inventory levels at the end of each period shown as black boxes. The ratios for biomass shipment and availability for Figure $4 \mathrm{a}, \mathrm{b}$, and $\mathrm{c}$ are 1, 0.875, and 0.944, respectively.

For example, consider the one-year harvesting, shipment and resulting inventory profiles at the harvesting site shown in Figure 4 . We assume that $100 \mathrm{kt}$ of biomass is available annually, $60 \%$ of it is harvested during period 1 and the remaining is harvested during period 2 . We further assume that biomass deteriorates at a 5\% rate per period. If biomass is directly shipped to the depot (Figure 4a), the inventory levels are zero and therefore the ratio (correction) is equal to $1\left(\delta_{i}=1\right)$. In the other extreme, all biomass is stored at the harvesting site and shipped at the end of the year $(t=4)$ as shown 
in Figure 4b. The highest inventory level, obtained at the end of period 2, is $92.5 \mathrm{kt}$ instead of $100 \mathrm{kt}$ due to biomass losses and the total shipment is $87.5 \mathrm{kt}$. In this case, the total losses are $12.5 \mathrm{kt}$ (100 - 87.5), and therefore $\delta_{i}=0.875$. If biomass is shipped at a constant rate throughout the year (Figure $4 \mathrm{c}$ ), then $\delta_{i}=0.944$. As we will see in Section 5, the loss rate is significantly smaller, leading to correction factors larger than 0.95 . Based on these calculations, we can choose the average value or a correction factor that corresponds to what we think is a reasonable inventory profile.

329 The flow, $F_{i^{\prime}, k, l, t}$, of intermediate $i^{\prime} \in \mathrm{I}^{\mathrm{ID}}$ from depot $k$ to biorefinery $l$ can be calculated based on the 330 flow from the harvesting site, $F_{i, j, k, t} \approx \delta_{i} \alpha_{i, j, t}$ according to our approximation, multiplied by the conversion factor $\eta_{i, i^{\prime}, m}$ (see Equations 3 and 4). Thus, after deterioration due to storage at the depot, the shipment of intermediate to the biorefinery can be approximated by $\zeta_{i^{\prime}} \eta_{i, i^{\prime}, m} \delta_{i} \alpha_{i, j, t}$ where $\zeta_{i^{\prime}}$ is the correction factor for intermediate storage losses at the depot. Similarly, $\zeta_{i^{\prime}}$ can be precalculated based on the ratio of intermediate shipment to the biorefinery and biomass consumption at the depot. The accuracy of the correction factors we use can be checked post optimization using the exact biomass/intermediate shipments obtained.

\subsection{Transportation Distance}

Based on the reformulation described in the previous subsection, the approximate shipments of biomass/intermediates is always non-zero $\left(\delta_{i} \alpha_{i, j, t}\right.$ or $\left.\zeta_{i^{\prime}} \eta_{i, i^{\prime}, m} \delta_{i} \alpha_{i, j, t}\right)$. Therefore, to correctly calculate transportation cost, these values should be multiplied by zero distance if the corresponding $\operatorname{arcs}\left(Z_{j, k} / Z_{j, l} / Z_{k, l}\right)$ are not selected. To address this, we disaggregate all distance components into actual, $\bar{D}_{j, k} / \bar{D}_{j, l} / \bar{D}_{k, l}$, and dummy, $\breve{D}_{j, k} / \breve{D}_{j, l} / \breve{D}_{k, l}$, distance variables:

$344 \quad D_{j, l}=\bar{D}_{j, l}+\breve{D}_{j, l}, \quad j, l$

$D_{k, l}=\bar{D}_{k, l}+\breve{D}_{k, l}, \quad k, l$

346 subject to the following variable upper bound constraints:

$347 \bar{D}_{j, k} \leq \tau_{j, k}^{\mathrm{U}} Z_{j, k}, \quad j, k$

$348 \quad \breve{D}_{j, k} \leq \tau_{j, k}^{\mathrm{U}}\left(1-Z_{j, k}\right), \quad j, k$

$\bar{D}_{j, l} \leq \tau_{j, l}^{\mathrm{U}} Z_{j, l}, \quad j, l$

$350 \quad \breve{D}_{j, l} \leq \tau_{j, l}^{\mathrm{U}}\left(1-Z_{j, l}\right), \quad j, l$

$\bar{D}_{k, l} \leq \tau_{k, l}^{\mathrm{U}} Z_{k, l}, \quad k, l$

353 where $\tau_{j, k}^{\mathrm{U}} / \tau_{j, l}^{\mathrm{U}} / \tau_{k, l}^{\mathrm{U}}$ are upper bounds on distance. When an arc is selected $(Z=1)$ the dummy 354 variables become zero via Equations. (46), (48), and (50). Therefore, the real distance equals to 
calculated distance $\left(\bar{D}_{j, k}=D_{j, k}\right)$. In contrast, $\bar{D}_{j, k}=0$ if the arc is not selected. For example, HS1 in Figure $5 \mathrm{a}$ is selected to provide biomass to the depot. $\bar{D}_{\mathrm{HS} 1, \mathrm{D} 1}$ (red line) is equal to $D_{\mathrm{HS} 1, \mathrm{D} 1}$ as $\breve{D}_{\mathrm{HS} 1, \mathrm{D} 1}$ is forced to be zero (Equation 46 ). When $Z=0, \bar{D}=0$, and $D$ will equal to $\breve{D}$. For instance, HS2 is not selected to provide biomass to the depot, $\bar{D}_{\mathrm{HS} 2, \mathrm{D} 1}=0$ and $\breve{D}_{\mathrm{HS} 2, \mathrm{D} 1}=D_{\mathrm{HS} 2, \mathrm{D} 1}$ (gray line). Note that only real variables, $\bar{D}_{j, k}, \bar{D}_{j, l}$, and $\bar{D}_{k, l}$ (color lines) will be considered in the cost calculation.

(a)

\section{Harvesting Site $j \circlearrowright$ Depot $k \hat{\sim}$ Biorefinery $l$ o Technology at depot $m$}

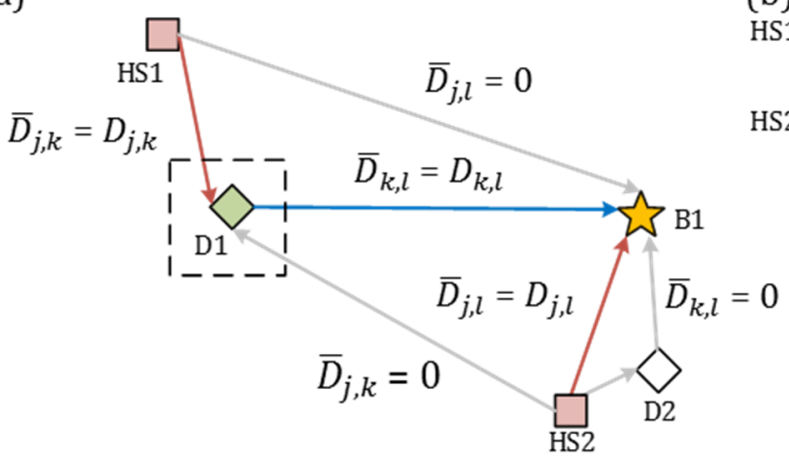

(b)

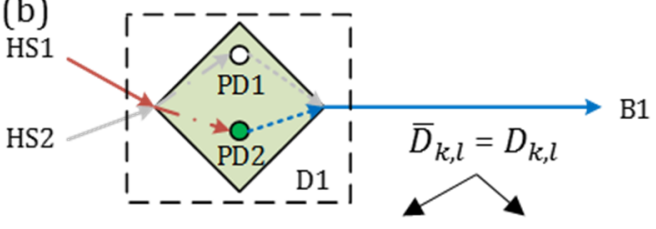

HS1

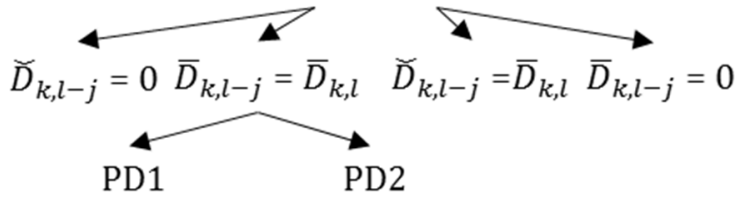

$$
\bar{D}_{k, l-j, m}=0 \quad \bar{D}_{k, l-j, m}=\bar{D}_{k, l-j}
$$

Figure 5: (a) Disaggregated real distances. Color/white boxes represent the SC node is selected and not selected, respectively. Real distances are shown in red and blue lines whereas dummy distances are shown in gray lines. (b) Disaggregated variables for $\bar{D}_{k, l}$.

364 As mentioned earlier, $F_{i^{\prime}, k, l, t}$ can be approximated by $\zeta_{i^{\prime}} \eta_{i, i^{\prime}, m} \delta_{i} \alpha_{i, j, t}$. In order to calculate transportation cost from $k$ to $l$ accurately, $\bar{D}_{k, l}$ is further disaggregated into $\bar{D}_{k, l-j, m}$ to trace the amount of biomass shipped from site $j$ and densified through technology $m$ :

$\bar{D}_{k, l}=\bar{D}_{k, l-j}+\widetilde{D}_{k, l-j}, \quad j, k, l$

$\bar{D}_{k, l-j}=\sum_{m \in \mathbf{M}^{\mathrm{PD}}} \bar{D}_{k, l-j, m}, j, k, l$

369 where disaggregated variables are subject to:

$\bar{D}_{k, l-j} \leq \tau_{k, l}^{\mathrm{U}} Z_{j, k}, \quad j, k, l$

371

$\breve{D}_{k, l-j} \leq \tau_{k, l}^{\mathrm{U}}\left(1-Z_{j, k}\right), \quad j, k, l$

372

$\bar{D}_{k, l-j, m} \leq \tau_{k, l}^{\mathrm{U}} U_{k, m}, \quad j, k, l, m \in \mathbf{M}^{\mathrm{PD}}$

373 In the example shown in Figure 5b, technology of PD2 is selected at the depot. For biomass shipped

374 from HS1, $\bar{D}_{\mathrm{D} 1, \mathrm{~B} 1-\mathrm{HS} 1, \mathrm{PD} 2}=\bar{D}_{\mathrm{D} 1, \mathrm{~B} 1-\mathrm{HS} 1}=\bar{D}_{\mathrm{D} 1, \mathrm{~B} 1}$. Note that $\bar{D}_{\mathrm{D} 1, \mathrm{~B} 1-\mathrm{HS} 1, \mathrm{PD} 1}=0$ since only one 375 technology is selected at the depot. On the other hand, for HS2, $\bar{D}_{\mathrm{D} 1, \mathrm{~B} 1-\mathrm{HS} 2}$ is equal to zero as no 376 biomass from HS2 is shipped to D1. 
Based on the reformulations introduced in the previous subsections, transportation cost can be calculated based on the total shipments, and the latter can be treated as parameters, thereby resulting in a linear transportation cost estimation:

\section{$383 \quad 4.4$ Tightening Constraints}

384 The total amount of biomass shipped from a harvesting site to a depot is bounded by multiples of the 385 total availability of biomass and the selection binary for $\operatorname{arc} j \rightarrow k$.

$386 \sum_{i, t} \pi_{i} \alpha_{i, j, t} Z_{j, k} \leq \sum_{i, t} F_{i, j, k, t} \leq \sum_{i, t} \alpha_{i, j, t} Z_{j, k}, \quad j, k$

387 where $\pi_{i}$ is the correction factor based on the worst case scenario. For example, in Figure 4, the upper 388 bound is set to $100 \mathrm{kt}$. The worst case scenario is when all biomass is stored at the harvesting site 389 and shipped at the end of the year (Figure $4 \mathrm{~b}$ ). Therefore, $\pi_{i}=0.875$ resulting in a lower bound of $390 \quad 87.5 \mathrm{kt}$.

391 Similarly, we can write variable lower/upper bounding constraints for the total amount of biomass 392 shipped to a biorefinery:

$\sum_{i, t} \pi_{i} \alpha_{i, j, t} Z_{j, l} \leq \sum_{i, t} F_{i, j, l, t} \leq \sum_{i \in \mathbf{I}, t} \alpha_{i, j, t} Z_{j, l}, \quad j, l$

394 The total shipments out of a harvesting site can also be variable lower/upper bounded as follows:

$\sum_{i, t} \pi_{i} \alpha_{i, j, t} W_{j} \leq \sum_{i, k, t} F_{i, j, k, t}+\sum_{i, l, t} F_{i, j, l, t} \leq \sum_{i, t} \alpha_{i, j, t} W_{j}, \quad j$

396 Finally, the lower bounds of transportation distance are added into the model and given by:

$397 \tau_{j, l}^{\mathrm{L}} \leq D_{j, l}, \quad j, l$

$398 \tau_{j, l}^{\mathrm{L}} Z_{j, l} \leq \bar{D}_{j, l}, \quad j, l$

399 where $\tau_{j, l}^{\mathrm{L}}$ is minimum distance between $\operatorname{arc} j$ and $l$, and it can be predetermined from the minimum 400 distance between harvesting site and the vertices of the potential biorefinery location.

\section{$401 \quad 4.5$ Relative Error Calculation}

402 Based on the biomass shipments obtained from the optimal/best solution, we can post-calculate the 403 "real" TAC and then the relative error:

$404 \%$ error $=\frac{T A C^{\text {real }}-T A C^{\text {approx }}}{T A C^{\text {real }}} \times 100 \%$

405 where $T A C^{\text {approx }}$ is the approximate value and $T A C^{\text {real }}$ is the real value. 


\section{Applications}

407 We present two examples based on the same region, Southern Wisconsin. We consider two types of 408 biomass: corn stover (CS) and switchgrass (SG), both of which are abundant in the selected region. 409 The availability of biomass is extracted from the US Department of Agriculture [43] and the Energy 410 Biosciences Institute [44]. Candidate biorefinery locations were restricted to cities with populations 411 between 3,000 and 10,000 in 2014 for a maximum of one biorefinery per county [45] and total 412 biomass availability more than 120,000 ton per year. The latitude and longitude of harvesting sites 413 and potential biorefineries are converted to Cartesian coordinates based on the Rhumb Line concept 414 [41]. Infeasible areas due to geographical impediment (e.g., lakes, reserved forests) are excluded from 415 the studied region during preprocessing. The rectilinear distance measurement method is used to 416 estimate the distance. Both CS and SG are harvested at the site during summer and fall. We consider 417 four time periods (spring, summer, fall and winter).

418 Collected CS and SG are sent for cutting and shredding, windrowing and baling prior to transporting 419 to the depot/biorefinery. The capacity of the depot is set between 100 and 200 dry $\mathrm{t} / \mathrm{d}$. Economics of 420 larger depot may not improve as it costs more to transport the biomass at a larger distance. We 421 consider two technologies at the depot. In the first technology (DD), CS and SG are dried, densified, 422 and pelletized directly without any chemical or physiochemical pretreatment. CS pellets (CSP) and 423 SG pellets (SGP) are the intermediates produced by the technology DD. In the second technology 424 (AFEXDD), CS and SG are pretreated via ammonia fiber explosion prior to densification. CS AFEX 425 pellet (CSAP) and SG AFEX pellet (SGAP) are produced by the technology AFEXDD. At the biorefinery, 426 we consider one pretreatment technology, AFEX; and two conversion technologies, simultaneous 427 saccharification and fermentation (SSCF) and separate hydrolysis and fermentation (SHCF). 428 Untreated biomass and intermediates (CS, SG, CSP and SGP) are pretreated via AFEX. The pretreated 429 biomass and intermediate (CSA and SGA) produced at the biorefinery, CSAP, and SGAP are further 430 processed in either SSCF or SHCF for ethanol production.

431 It is assumed that the material loss factors of CS/SG, CSP/SGP, and CSAP/SGAP for on-site storage are $4321.5 \%, 0.7 \%$ and $0.2 \%$ every three months, respectively. Based on the availability of biomass at each 433 period, the correction factors $\delta_{i}$ and $\zeta_{i^{\prime}}$ are precalculated. The ranges of correction factor for 434 compounds are $\delta_{\mathrm{CS}} / \delta_{\mathrm{SG}} \in[0.961,1], \zeta_{\mathrm{CSP}} / \zeta_{\mathrm{SGP}} \in[0.982,1]$, and $\zeta_{\mathrm{CSAP}} / \zeta_{\mathrm{SGAP}} \in[0.995,1]$. In this 435 study, it is assumed that the correction factors are the average values: $\delta_{\mathrm{CS}} / \delta_{\mathrm{SG}}=0.981, \zeta_{\mathrm{CSP}} / \zeta_{\mathrm{SGP}}=$ 4360.991 , and $\zeta_{\mathrm{CSAP}} / \zeta_{\mathrm{SGAP}}=0.997$. We further assume that depots and biorefineries operate 85 days 437 during every period. An annual discount rate of $10 \%$ and a project lifetime of 25 years are considered 438 for the calculation of the annualized capital cost. The detailed descriptions of the cost and conversion 439 data are provided in the Supplementary Material [29,32,46-51]. Note that all costs are indexed to 4402014 dollars and calculated based on dry mass basis. We present two examples in next sub-sections. 


\subsection{Example 1}

442 We consider a biofuel SC in South Central Wisconsin (215 km $\times 120 \mathrm{~km})$ that includes Columbia, 443 Dane, Dodge, Grant, Green, Iowa, Jefferson, Lafayette, Richland, Rock, and Sauk counties. Based on 444 the preliminary screening, four potential biorefinery locations (Oregon Village, Columbus, Mayville 445 and Beloit) are identified. For this example, we identify only two biorefineries which are located near 446 to the center of studied region: Oregon Village and Columbus. At most three depots can be installed 447 in the studied region. We discretize these 11 counties into 64 cells (Figure 6). The minimum ethanol 448 demand is assumed to be 340 million L/y. Our objective is to minimize TAC. The resulting MILP 449 model, which consists of 431 binary variables and 8,030 continuous variables, was implemented in 450 GAMS [52] and solved using CPLEX.

451 The best solution was found after 3,405 CPU seconds with an optimality gap equal to $0.43 \%$. The 452 minimum total annual cost obtained is \$ 251.44 million. Figure 6 illustrates the optimal locations of 453 the depots and the biorefinery. As shown, for the harvesting sites (light red regions) which are 454 located near the biorefinery, direct biomass shipments are chosen. As the distance between the 455 harvesting sites and the biorefinery increases (e.g., sites located at light blue regions), the 456 transportation cost of direct biomass shipment increases and at some point becomes larger than the 457 depot cost (capital and operating) plus the intermediate transportation cost. Thus, depots at the light 458 blue regions are selected to covert biomass to a higher bulk density pellet. Two depots employing DD 459 technologies are selected in Grant and Dodge counties. Specifically, the depots are installed at Far 460 East Wyalusing and North East Iron Ridge with the capacities of 113 and $109 \mathrm{t} / \mathrm{d}$, respectively. The 461 biorefinery with AFEX and SHCF is installed at West Oregon village. Note that only half of the available 462 biomass in Richland County is utilized.

463 The total biomass losses at the harvesting sites before shipped to the depots and the biorefinery are $4641,387 \mathrm{t} / \mathrm{y}$ and 19,837 t/y, respectively which yield a total shipments:availability ratio equal to 0.982 465 and 0.983 , respectively. At the depot, the overall loss is $785.3 \mathrm{t} / \mathrm{y}$ which leads to a ratio equal to 0.990. 466 Note that the ratios calculated based on the actual shipment (0.982, 0.983, and 0.990) are very similar 467 to the correction factors that were used $(0.981,0.991,0.997)$. As a result, the transportation cost 468 based on the calculated flows (USD 30.08 million) is less than $0.1 \%$ higher than the linearly 469 approximated transportation cost (USD 30.06 million). Therefore, the "real" TAC is $\$ 251.46$ million, 470 which is only $0.01 \%$ higher than the TAC obtained by the proposed model. 


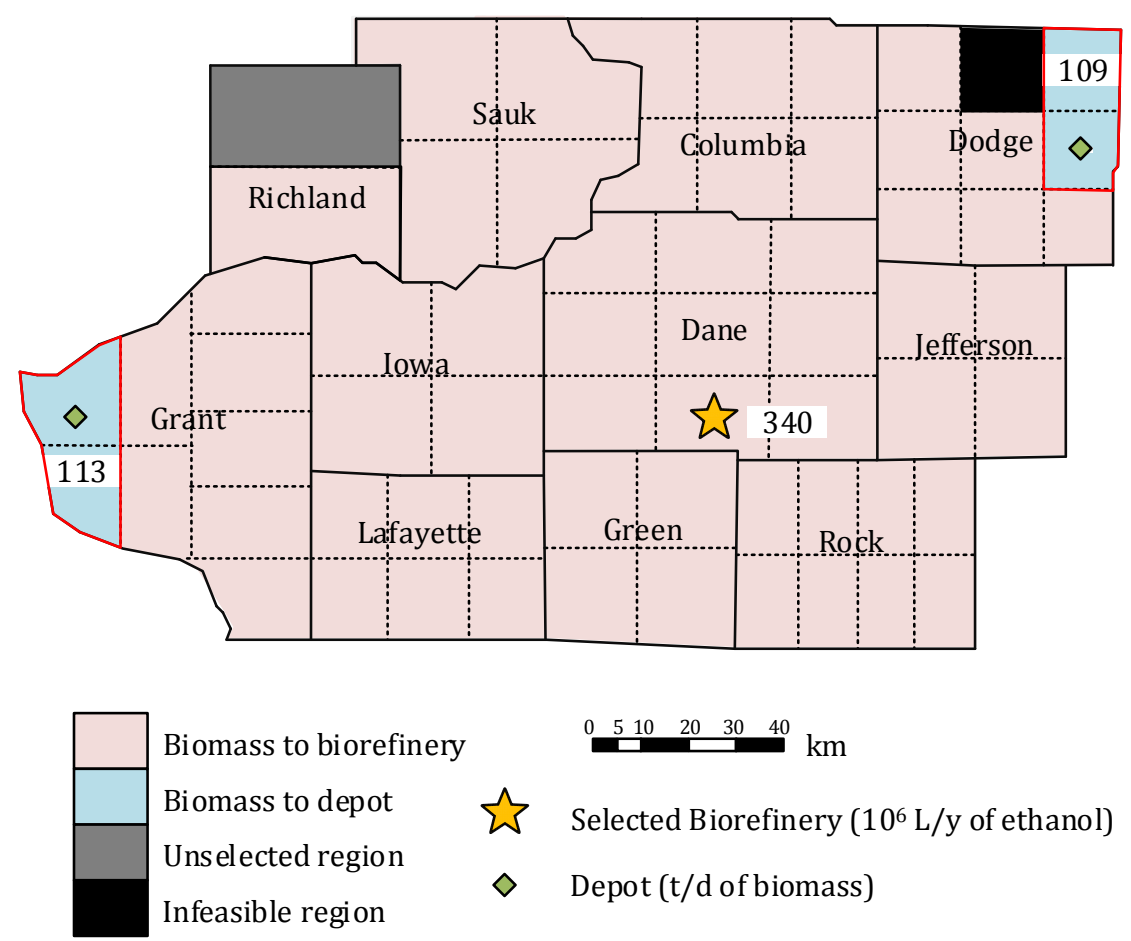

471

472

473

474

475

476

477

478

479

480

481

482

483

Figure 6: Best SC configuration for Example 1

For comparison purposes, we extended the MINLP model [30] to account for variable biorefinery locations and solved Example 1 using a resource limit of 15,000 CPU seconds. The best solution obtained using the extended MINLP model had a TAC of \$ 251.91 million (relative gap of 1.8\%) which is worse than the solution obtained by the proposed model. The higher TAC was primarily due to the selection of three depots instead of two.

\subsection{Example 2}

We consider a biofuel SC in South Wisconsin $(300 \mathrm{~km} \times 200 \mathrm{~km})$ that includes 32 counties (Adams, Calumet, Columbia, Crawford, Dane, Dodge, Fond du Lac, Grant, Green, Green Lake, Iowa, Jefferson, Juneau, Kenosha, La Crosse, Lafayette, Manitowoc, Marquette, Milwaukee, Monroe, Ozaukee, Racine, Richland, Rock, Sauk, Sheboygan, Vernon, Walworth, Washington, Waukesha, Waushara, and Winnebago). We divide the area into 194 cells and each cell has 9,500 to 13,000 t/y of CS and SG except for the counties with small biomass availability (e.g., Milwaukee and Ozaukee). The minimum ethanol demand is assumed to be 560 million L/y. Based on the preliminary screening, four potential biorefinery locations (Oregon Village, Columbus, Mayville and Beloit) are identified. In this example, Beloit is not included as a potential biorefinery location as it would lead to large transportation cost since it is near the state line between Wisconsin and Illinois. Our objective is to minimize TAC. To reduce the computational requirements, we partition the original problem into subproblems with fixed number of SC nodes. Each subproblem can represented by a duplet, D.B, where D is the number of depots, and B denotes the selected biorefineries. For example, in instance D3.BO we minimize the 
493 for depots: zero to nine depots (D0 to D9); and three options for biorefineries: Oregon Village (BO),

494 Columbus (BC), and Mayville (BM). A total of 30 configurations is generated based on these options.

495 The best solution was found with eight depots and a biorefinery installed in Columbus (D8.BC). The

496 relative gap is $1.06 \%$. The minimum TAC is $\$ 403.29$ million. Figure 7 illustrates the optimal locations

497 of the depots and the biorefinery. As shown, there are two depots to be installed in Grant County and

498 one depot in six other counties (La Crosse, Monroe, Vernon, Lafayette, Calumet, and Manitowoc). For

499 all depots, DDs with different capacities are installed. The area of biomass shipped to the depot is

500 bounded by a red line. Part of biomass in La Crosse, Monroe and Grant counties are not selected for

501 ethanol production. The biorefinery is located at South West Columbus. At the biorefinery, both AFEX

502 and SSCF are selected for bioethanol production. Similar to Example 1, the TAC is recalculated (USD

503403.48 million) based on the flow obtained in the best solution. The calculated TAC is only $0.05 \%$

504 higher than the approximate value obtained using the proposed model.

505 Figure 8 shows biomass availability, shipment, and inventory profiles at two different harvesting 506 sites (Dodge and Lafayette counties). The distance between the harvesting sites and the biorefinery 507 are 17 and $155 \mathrm{~km}$, respectively. When the destination is close (Figure 8a), both CS and SG are 508 shipped immediately. As the distance increases (Figure 8b), CS is stored (red dashed line) and only 509 shipped during period 1 . This is because as the distance increases, transportation cost increases, 510 which means that it is more profitable to store for longer periods of time the biomass that was 511 harvested further away because this will lead to lower total transportation cost. Please note that SG 512 is sent for ethanol production directly during periods 2 and 3 in order to achieve minimum ethanol 513 demand for each period.

514 Figure 9a shows biomass consumption (positive red bar) and production of pellets (blue bar) at the 515 depot located at Layette County. We observe that production consumption is constant the year. This 516 production profile leads to the smallest capital investment (for the same total production). Pellets 517 are stored (blue dashed line) and shipped to the biorefinery during period $1(50,000 \mathrm{t})$.

518 The consumption and production levels at the biorefinery are shown in Figure 9b. At the biorefinery, 519 biomass and pellets are converted to ethanol at $t=1$ whereas only biomass is used for ethanol 520 production during the remaining periods. Note that no ethanol is stored (i.e., production = sales) 521 which means that the biorefinery operates at full capacity during the entire year to maximize the 522 utilization of expensive assets. 


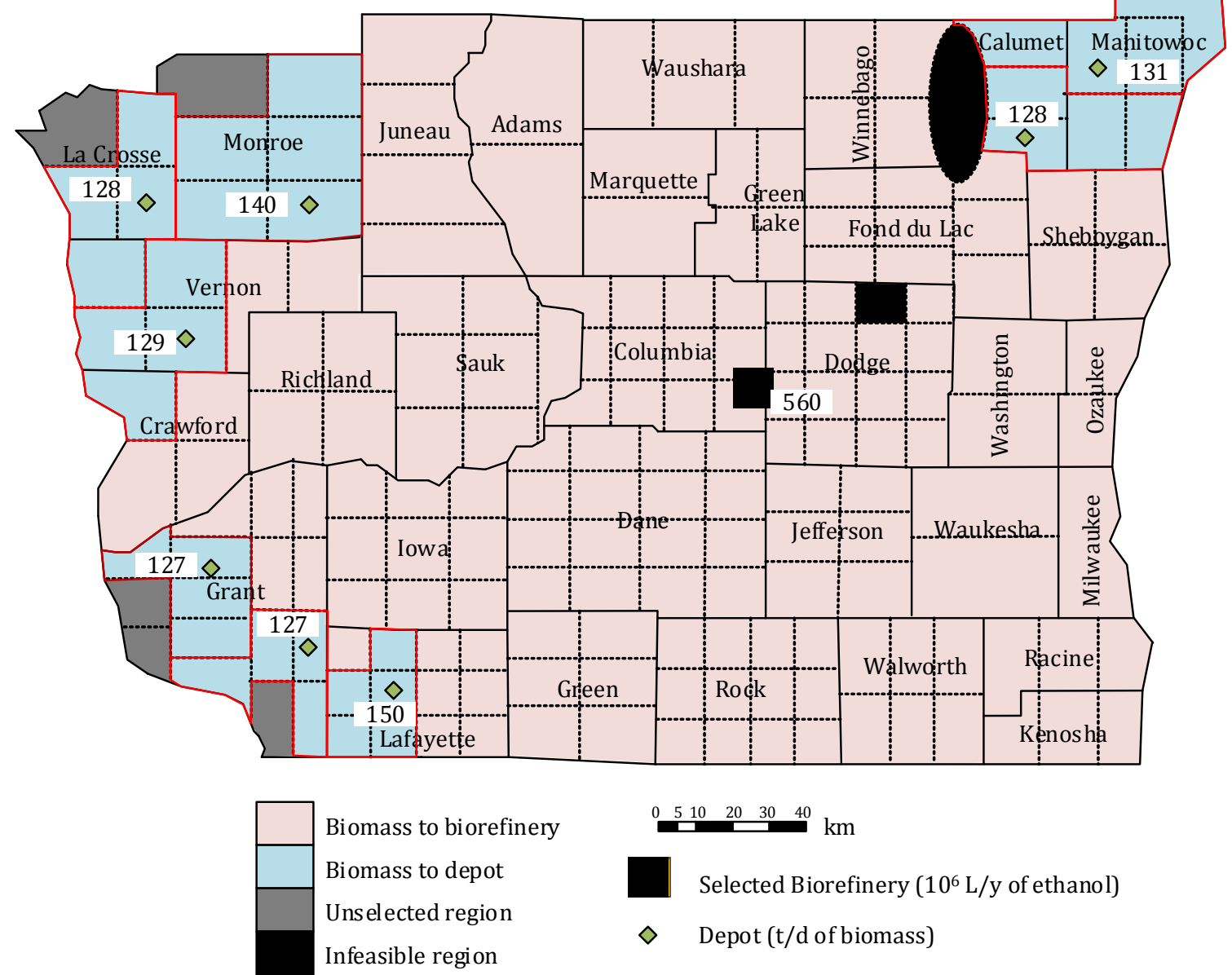

Figure 7: Best SC configuration for Example 2
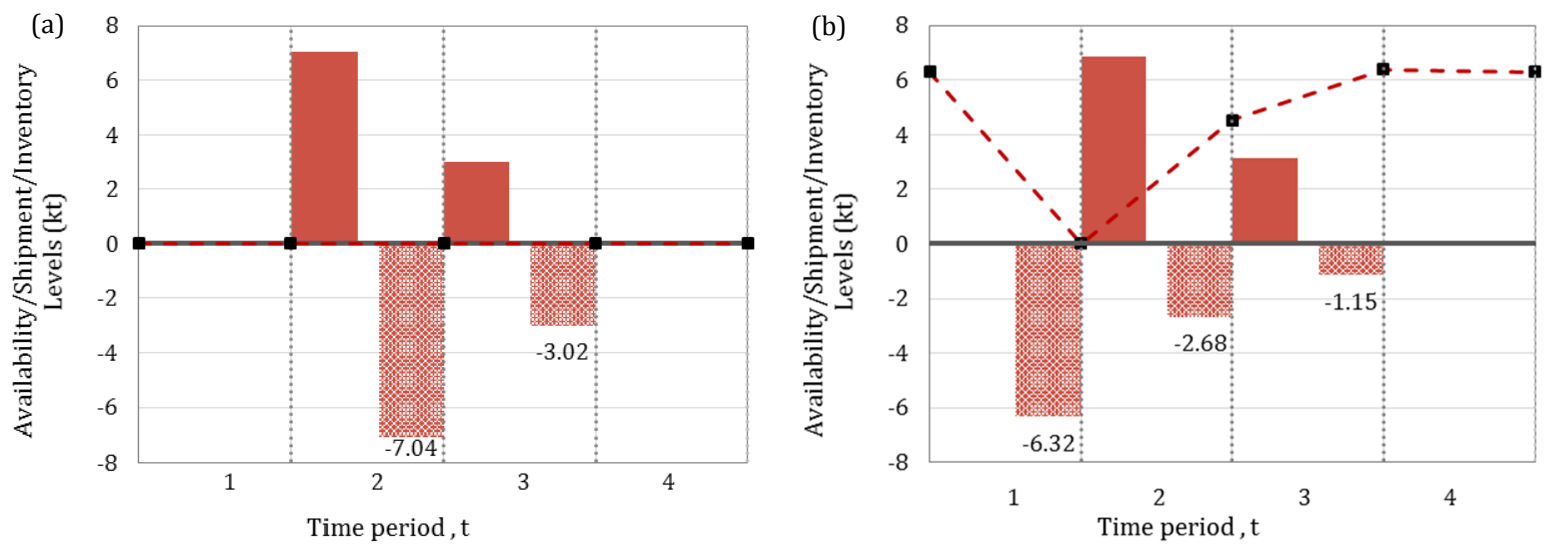

Figure 8: Consumption, production, shipment, and inventory levels at the harvesting sites. Inventory of biomass is shown with red dashed line. Net biomass accumulation (availability - shipments) at the harvesting site (red bar). 
(a)

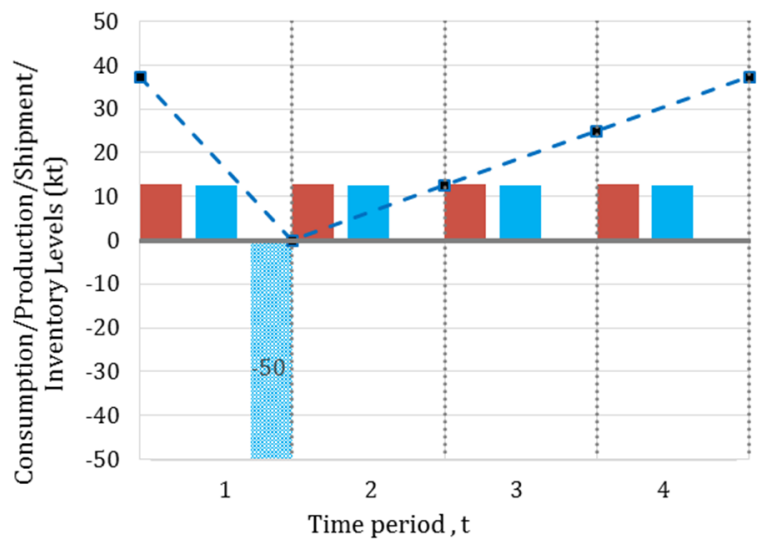

(b) 560

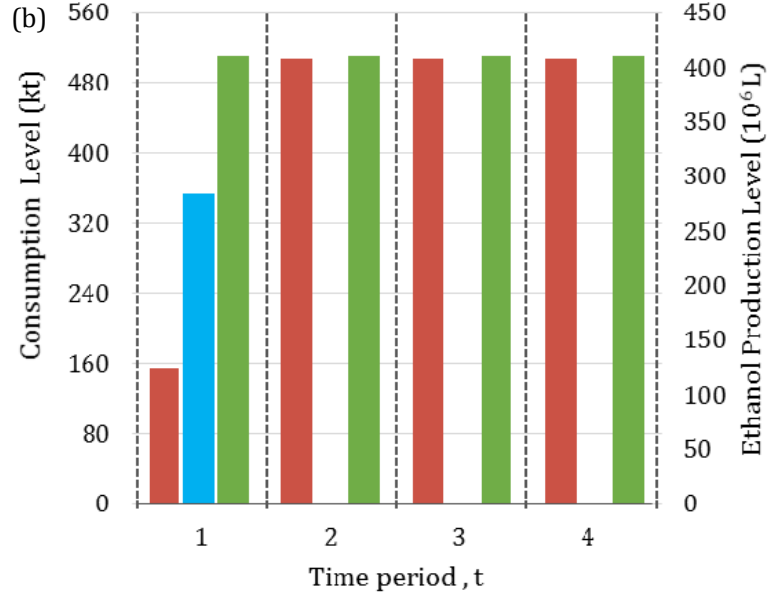

\section{3}

534

\section{Acknowledgements}

543 This work was funded by the DOE Great Lakes Bioenergy Research Center (DOE BER Office of Science 544 DE-FC02-07ER64494).

Figure 9: Consumption and production levels at (a) the depot and (b) the biorefinery. Inventory of pellet is shown with blue dashed line. Biomass (red bar), net pellet accumulation (production - shipments) (blue bar), and ethanol (green bar) are shown.

\section{Conclusions}

The goal of this paper was to propose an MILP model that allows us to address larger-scale SC problems that are common in real-world applications. The proposed MILP model treats the locations of depots and biorefineries as optimization decisions. We proposed an approximation method for approximating the amount of biomass and intermediates of shipment. The correction factors can be precalculated based on the availability of biomass at certain period and its deterioration factor. We also reformulated the transportation distance and added tightening constraints in the proposed model. Furthermore, the proposed model can be revised to handle different features and practical considerations. 


\section{Nomenclature}

547 Sets

$548 \quad i \in \mathbf{I}$

$549 j \in \mathbf{J}$

Compounds

$550 \quad k \in \mathbf{K}$

Harvesting sites

$551 l \in \mathbf{L}$

Depots

$552 m \in \mathbf{M}$

Biorefineries

$553 \quad o \in \mathbf{0}$

Technologies

$554 t \in \mathbf{T}$

Vertices

\section{Subsets}

$556 \quad$ I $^{\mathrm{F} / \mathrm{ID} / \mathrm{IB} / \mathrm{P} / \mathrm{B}}$

557

558

$\mathbf{M}^{\mathrm{PD} / \mathrm{PB} / \mathrm{CB}}$

559

Time periods

560

561

562

$563 \gamma_{i, t}$

$564 \delta_{i} / \zeta_{i}$

$565 \quad \eta_{i, i^{\prime}, m}$

$566 \iota_{i}$

$567 \quad \kappa_{i}^{\mathrm{F}} / \kappa_{i}^{\mathrm{V}}$

$568 \lambda_{i}$

$569 \mu_{m}$

$570 v$

$571 \quad x_{j} / y_{j}$

$572 x_{l, o} / y_{l, o}$
Biomass feedstocks; intermediates produced at depots; intermediates produced at biorefineries; products; by-products

Pretreatment and/or densification technologies at depot; pretreatment technologies at biorefinery; conversion technologies at biorefinery

Availability of compound $i$ at harvesting site $j$ during period $t(\mathrm{t})$

Lower/upper bound on product demand during period $t(\mathrm{t})$

Material loss factor of compound $i$ during period $t$

Correction factor for compound $i$

Conversion coefficient of compound $i$ to compound $i$ ' by technology $m$

Unit inventory cost of compound $i(\$ / \mathrm{t})$

Fixed/variable unit transportation cost of compound $i(\$ / \mathrm{t}$ or $\$ / \mathrm{t}-\mathrm{km})$

Unit cost of compound $i(\$ / t)$

Unit production cost of technology $m(\$ / t)$

Capital recovery factor

Predefined $\mathrm{x}-/ \mathrm{y}$-Cartesian coordinate of harvesting site $j(\mathrm{~km})$

Coordinates of the vertices of a potential biorefinery $l(\mathrm{~km})$ location

\section{Binary Variables}

$574 \quad U_{k, m} / U_{l, m} \quad=1$ if technology $m$ at depot $k /$ biorefinery $l$ is selected

$575 W_{j} / W_{k} / W_{l} \quad=1$ if harvesting site $j /$ depot $k /$ biorefinery $l$ is selected

$576 Z_{j, k} / Z_{j, l} / Z_{k, l} \quad=1$ if transportation along $\operatorname{arc} j \rightarrow k / j \rightarrow l / k \rightarrow l$ is selected

\section{Continuous Nonnegative Variables}

$578 \quad \breve{D}_{j, k} / \breve{D}_{j, l} / \breve{D}_{k, l}$

Disaggregated dummy variables for $D_{j, k} / D_{j, l} / D_{k, l}(\mathrm{~km})$

$579 \quad \bar{D}_{j, k} / \bar{D}_{j, l} / \bar{D}_{k, l}$

Disaggregated real variables for $D_{j, k} / D_{j, l} / D_{k, l}(\mathrm{~km})$ 
$591 \quad G_{i, l, m, t}^{\mathrm{F}-\mathrm{PB}}$

592

593

$G_{i, k, m, t}^{\mathrm{F}-\mathrm{PD}}$

$G_{i, l, m, t}^{\mathrm{IB}-\mathrm{CB}}$

\section{$L_{i, l, t}$}

$P_{i, k, m, t}^{\mathrm{ID}-\mathrm{PD}}$

$$
P_{i, l, m, t}^{\mathrm{IB}-\mathrm{PB}}
$$

$P_{i, l, m, t}^{\mathrm{IPB}-\mathrm{CB}}$

$$
Q_{k, m} / Q_{l, m}
$$

$7 x_{k}^{*} / x_{l}^{*}$

$y_{k}^{*} / y_{l}^{*}$

TAC
$C^{\mathrm{CAPEX}} / C_{k, m}^{\mathrm{CAPEX}} / C_{l, m}^{\mathrm{CAPEX}}$

$C_{t}^{\text {FEED/INV/PROD/TRANS }}$

$D_{j, k} / D_{j, l} / D_{k, l}$

$F_{i, j, k, t} / F_{i, j, l, t} / F_{i, k, l, t}$

$G_{i, l, m, t}^{\mathrm{ID}-\mathrm{PB}} / G_{i, l, m, t}^{\mathrm{ID}-\mathrm{CB}}$$$
S_{i, j, t} / S_{i, k, t} / S_{i, l, t}
$$

$\Delta x_{j, k} / \Delta x_{j, l} / \Delta x_{k, l}$

$\Delta y_{j, k} / \Delta y_{j, l} \Delta y_{k, l}$
Disaggregated real variable for $\bar{D}_{k, l}$ with respect to harvesting site $j$ and technology $m(\mathrm{~km})$

Disaggregated real/dummy variables for $\bar{D}_{k, l}$ with respect to harvesting site $j(\mathrm{~km})$

Total capital cost; capital cost of technology $m$ at depot $k /$ biorefinery $l(\$)$

Feedstock/inventory/production/transportation costs during period $t(\$)$

Distance along arc $j \rightarrow k / j \rightarrow l / k \rightarrow l(\mathrm{~km})$

Amount of compound $i$ shipped along arc $j \rightarrow k / j \rightarrow l / k \rightarrow l$ during period $t$ $(\mathrm{t})$

Consumption level of compound $i \in \mathbf{I}^{\mathrm{F}}$ by technology $m \in \mathbf{M}^{\mathrm{PD}}$ at $\operatorname{depot} k$ during period $t .(\mathrm{t})$

Consumption level of compound $i \in \mathbf{I}^{\mathrm{F}}$ by technology $m \in \mathbf{M}^{\mathrm{PB}}$ at biorefinery $l$ during period $t(\mathrm{t})$

Consumption level of compound $i \in \mathbf{I}^{\mathrm{IB}}$ by technology $m \in \mathbf{M}^{\mathrm{CB}}$ at biorefinery $l$ during period $t(\mathrm{t})$

Consumption level of compound $i \in \mathbf{I}^{\mathrm{ID}}$ by technology $m \in \mathbf{M}^{\mathrm{PB}} / \mathbf{M}^{\mathrm{CB}}$ at biorefinery $l$ during period $t(\mathrm{t})$

Total sales of compound $i \in \mathbf{I}^{\mathrm{P}} \cup \mathbf{I}^{\mathrm{B}}$ from biorefinery $l$ during period $t(\mathrm{t})$ Production level of compound $i \in \mathbf{I}^{\mathrm{ID}}$ by technology $m \in \mathbf{M}^{\mathrm{PD}}$ at $\operatorname{depot} k$ during period $t(\mathrm{t})$

Production level of compound $i \in \mathbf{I}^{\mathrm{IB}}$ by technology $m \in \mathbf{M}^{\mathrm{PB}}$ at biorefinery $l$ during period $t(\mathrm{t})$

Production level of compound $i \in \mathbf{I}^{\mathrm{P}} / \mathbf{I}^{\mathrm{B}}$ by technology $m \in \mathbf{M}^{\mathrm{CB}}$ at biorefinery $l$ during period $t(\mathrm{t})$

Capacity level of technology $m$ at $\operatorname{depot} k /$ biorefinery $l(\mathrm{t})$

Inventory level of compound $i$ at SC node $j / k / l$ at the end of time period $t$ $(\mathrm{t})$

$x$-Cartesian coordinate of depot $k$ /biorefinery $l(\mathrm{~km})$

$y$-Cartesian coordinate of depot $k$ /biorefinery $l(\mathrm{~km})$

Horizontal distance along arc $j \rightarrow k / j \rightarrow l / k \rightarrow l(\mathrm{~km})$

Vertical distance along $\operatorname{arc} j \rightarrow k / j \rightarrow l / k \rightarrow l(\mathrm{~km})$

Total annual cost $(\$ / y)$ 


\section{References}

613 [1] P. Beiter, 2014 Renewable Energy Data Book, 2015.

614 [2] IRENA, Renewable Energy Prospects: United States of America, REmap 2030 analysis, IRENA, 615 Abu Dhabi, 2015.

616 [3] H. An, W.E. Wilhelm, S.W. Searcy, Biofuel and petroleum-based fuel supply chain research: A literature review, Biomass and Bioenergy. 35 (2011) 3763-3774.

[4] B. Sharma, R.G. Ingalls, C.L. Jones, A. Khanchi, Biomass supply chain design and analysis: Basis, overview, modeling, challenges, and future, Renew. Sustain. Energy Rev. 24 (2013) 608-627.

[5] D. Yue, F. You, Fair profit allocation in supply chain optimization with transfer price and revenue sharing: MINLP model and algorithm for cellulosic biofuel supply chains, AIChE J. 60 (2014) 3211-3229.

[6] J.A. Elia, C.A. Floudas, Energy Supply Chain Optimization of Hybrid Feedstock Processes: A

[7] D.J. Garcia, F. You, Supply chain design and optimization: Challenges and opportunities, Comput. Chem. Eng. 81 (2015) 153-170.

[8] P. Daoutidis, W. Alex Marvin, S. Rangarajan, A.I. Torres, Engineering Biomass Conversion

[9] P. Daoutidis, A. Kelloway, W. Alex Marvin, S. Rangarajan, A.I. Torres, Process systems

[10] D.K.S. Ng, R.T.L. Ng, Applications of process system engineering in palm-based biomass

[11] J. Han, S. Murat Sen, J.S. Luterbacher, D.M. Alonso, J.A. Dumesic, C.T. Maravelias, Process systems engineering studies for the synthesis of catalytic biomass-to-fuels strategies, Comput. Chem. Eng. 81 (2015) 57-69.

[12] J. Kim, M.J. Realff, J.H. Lee, C. Whittaker, L. Furtner, Design of biomass processing network for biofuel production using an MILP model, Biomass and Bioenergy. 35 (2011) 853-871.

[13] J.A. Elia, R.C. Baliban, C.A. Floudas, B. Gurau, M.B. Weingarten, S.D. Klotz, Hardwood Biomass to Gasoline, Diesel, and Jet Fuel: 2. Supply Chain Optimization Framework for a Network of Thermochemical Refineries, Energy \& Fuels. 27 (2013) 4325-4352.

[14] F.E. Andersen, M.S. Díaz, I.E. Grossmann, Multiscale strategic planning model for the design of integrated ethanol and gasoline supply chain, AIChE J. 59 (2013) 4655-4672.

[15] L. Čuček, M. Martín, I.E. Grossmann, Z. Kravanja, Multi-period synthesis of optimally integrated biomass and bioenergy supply network, Comput. Chem. Eng. 66 (2014) 57-70.

[16] A. De Meyer, D. Cattrysse, J. Van Orshoven, Considering biomass growth and regeneration in the optimisation of biomass supply chains, Renew. Energy. 87 (2016) 990-1002.

[17] L. Čuček, P.S. Varbanov, J.J. Klemeš, Z. Kravanja, Total footprints-based multi-criteria optimisation of regional biomass energy supply chains, Energy. 44 (2012) 135-145.

[18] S. Giarola, A. Zamboni, F. Bezzo, Environmentally conscious capacity planning and technology selection for bioethanol supply chains, Renew. Energy. 43 (2012) 61-72. 
[19] A.M. El-Halwagi, C. Rosas, J.M. Ponce-Ortega, A. Jiménez-Gutiérrez, M.S. Mannan, M.M. ElHalwagi, Multiobjective optimization of biorefineries with economic and safety objectives, AIChE J. 59 (2013) 2427-2434.

[20] J.E. Santibañez-Aguilar, J.B. González-Campos, J.M. Ponce-Ortega, M. Serna-González, M.M. ElHalwagi, Optimal planning and site selection for distributed multiproduct biorefineries involving economic, environmental and social objectives, J. Clean. Prod. 65 (2014) 270-294.

[21] J. Kim, M.J. Realff, J.H. Lee, Optimal design and global sensitivity analysis of biomass supply chain networks for biofuels under uncertainty, Comput. Chem. Eng. 35 (2011) 1738-1751.

[22] I. Awudu, J. Zhang, Stochastic production planning for a biofuel supply chain under demand and price uncertainties, Appl. Energy. 103 (2013) 189-196.

[23] S. Bairamzadeh, M.S. Pishvaee, M. Saidi-Mehrabad, A multi-objective robust possibilistic programming approach to sustainable bioethanol supply chain design under multiple uncertainties, Ind. Eng. Chem. Res. 55 (2016) 237-256.

[24] K. Tong, J. Gong, D. Yue, F. You, Stochastic Programming Approach to Optimal Design and Operations of Integrated Hydrocarbon Biofuel and Petroleum Supply Chains, ACS Sustain. Chem. Eng. 2 (2014) 49-61.

[25] W. Alex Marvin, L.D. Schmidt, P. Daoutidis, Biorefinery Location and Technology Selection Through Supply Chain Optimization, Ind. Eng. Chem. Res. 52 (2013) 3192-3208.

[26] F. Zhang, D. Johnson, M. Johnson, D. Watkins, R. Froese, J. Wang, Decision support system integrating GIS with simulation and optimisation for a biofuel supply chain, Renew. Energy. 85 (2016) 740-748.

[27] F. You, B. Wang, Life Cycle Optimization of Biomass-to-Liquid Supply Chains with DistributedCentralized Processing Networks, Ind. Eng. Chem. Res. 50 (2011) 10102-10127.

[28] P.L. Eranki, B.E. Dale, Comparative life cycle assessment of centralized and distributed biomass processing systems combined with mixed feedstock landscapes, GCB Bioenergy. 3 (2011) 427-438.

[29] S. Kim, B.E. Dale, Comparing alternative cellulosic biomass biorefining systems: Centralized versus distributed processing systems, Biomass and Bioenergy. 74 (2015) 135-147.

[30] R.T.L. Ng, C.T. Maravelias, Design of Cellulosic Ethanol Supply Chains with Regional Depots, Ind. Eng. Chem. Res. 55 (2016) 3420-3432.

[31] P.L. Eranki, B.D. Bals, B.E. Dale, Advanced regional biomass processing depots: a key to the logistical challenges of the cellulosic biofuel industry, Biofuels, Bioprod. Biorefining. 5 (2011) 621-630.

[32] P. Lamers, M.S. Roni, J.S. Tumuluru, J.J. Jacobson, K. Cafferty, J.K. Hansen, et al., Technoeconomic analysis of decentralized biomass processing depots, Bioresour. Technol. 194 (2015) 205-213.

[33] H.K. Murnen, V. Balan, S.P.S. Chundawat, B. Bals, L. da C. Sousa, B.E. Dale, Optimization of Ammonia Fiber Expansion (AFEX) Pretreatment and Enzymatic Hydrolysis of Miscanthus X giganteus to Fermentable Sugars, Biotechnol. Prog. 23 (2007) 846-850.

[34] B.C. Saha, L.B. Iten, M.A. Cotta, Y.V. Wu, Dilute acid pretreatment, enzymatic saccharification and fermentation of wheat straw to ethanol, Process Biochem. 40 (2005) 3693-3700. 
[35] G. Banerjee, S. Car, J.S. Scott-Craig, D.B. Hodge, J.D. Walton, Alkaline peroxide pretreatment of corn stover: effects of biomass, peroxide, and enzyme loading and composition on yields of glucose and xylose., Biotechnol. Biofuels. 4 (2011) 16.

[36] D. Humbird, R. Davis, L. Tao, C. Kinchin, D. Hsu, A. Aden, et al., Process Design and Economics for Biochemical Conversion of Lignocellulosic Biomass to Ethanol. Dilte-Acid Pretreatment and Enzymatic Hydrolysis of Corn Stover, NREL. (2011).

[37] A. Dutta, S.D. Phillips, Thermochemical Ethanol via Direct Gasification and Mixed Alcohol Synthesis of Lignocellulosic Biomass, National Renewable Energy Laboratory (NREL), Golden, CO, 2009.

[38] A.M. Niziolek, O. Onel, J.A. Elia, R.C. Baliban, X. Xiao, C.A. Floudas, Coal and Biomass to Liquid Transportation Fuels: Process Synthesis and Global Optimization Strategies, Ind. Eng. Chem. Res. 53 (2014) 17002-17025.

[39] J.S. Luterbacher, J.M. Rand, D.M. Alonso, J. Han, J.T. Youngquist, C.T. Maravelias, et al., Nonenzymatic Sugar Production from Biomass Using Biomass-Derived $\gamma$-Valerolactone, Science (80-. ). 343 (2014) 277-280.

[40] J. Han, J.S. Luterbacher, D.M. Alonso, J.A. Dumesic, C.T. Maravelias, A lignocellulosic ethanol strategy via nonenzymatic sugar production: process synthesis and analysis., Bioresour. Technol. 182 (2015) 258-66.

[41] D.H. Maling, Coordinate Systems and Map Projections, Second Edi, Pergamon Press, Elmsford, New York, 1992.

[42] C.B. Barber, D.P. Dobkin, H. Huhdanpaa, The quickhull algorithm for convex hulls, ACM Trans. Math. Softw. 22 (1996) 469-483.

[43] USDA Census Of Agriculture, 2012 Census Publications, (2012). http://www.agcensus.usda.gov/Publications/2012/ (accessed November 12, 2015).

[44] Energy Biosciences Institute, Biofuel Ecophysiological Traits and Yields Database, (2015). https://www.betydb.org/ (accessed November 12, 2015).

[45] W. Alex Marvin, L.D. Schmidt, S. Benjaafar, D.G. Tiffany, P. Daoutidis, Economic Optimization of a Lignocellulosic Biomass-to-Ethanol Supply Chain, Chem. Eng. Sci. 67 (2012) 68-79.

[46] S. Brechbill, W.E. Tyner, The Economics of Renewable Energy: Corn Stover and Switchgrass, Purdue Ext. (2008). https://www.extension.purdue.edu/extmedia/EC/RE-3-W.pdf (accessed July 6, 2015).

[47] S. Sokhansanj, S. Mani, A. Turhollow, A. Kumar, D. Bransby, L. Lynd, et al., Large-scale production, harvest and logistics of switchgrass (Panicum virgatum L.) - current technology and envisioning a mature technology, Biofuels, Bioprod. Biorefining. 3 (2009) 124-141.

[48] D. Humbird, R. Davis, L. Tao, C. Kinchin, D. Hsu, A. Aden, et al., Process Design and Economics for Biochemical Conversion of Lignocellulosic Biomass to Ethanol: Dilute-Acid Pretreatment and Enzymatic Hydrolysis of Corn Stover, National Renewable Energy Laboratory (NREL), Golden, CO, 2011.

[49] A. Aden, M. Ruth, K. Ibsen, J. Jechura, K. Neeves, J. Sheehan, et al., Lignocellulosic Biomass to Ethanol Process Design and Economics Utilizing Co-Current Dilute Acid Prehydrolysis and Enzymatic Hydrolysis for Corn Stover, National Renewable Energy Laboratory (NREL), Golden, CO, 2002. 
734
[50] B. Bals, C. Wedding, V. Balan, E. Sendich, B. Dale, Evaluating the impact of ammonia fiber expansion (AFEX) pretreatment conditions on the cost of ethanol production, Bioresour. Technol. 102 (2011) 1277-1283.

[51] K.E. Ileleji, S. Sokhansanj, J.S. Cundiff, Farm-Gate to Plant-Gate Delivery of Lignocellulosic Feedstocks from Plant Biomass for Biofuel Production, in: Biofuels from Agric. Wastes Byprod., Wiley-Blackwell, 2010: pp. 117-159.

[52] GAMS Development Corporation, General Algebraic Modeling System (GAMS) Release 24.4.5, Washington, DC, USA, 2015. 